\title{
Arbor
}

\section{Envejecimiento y familia}

\section{María Teresa Bazo}

Arbor CLXXVIII, 702 (Junio 2004), 323-344 pp.

\section{Envejecimiento}

El envejecimiento de la población es resultado de la disminución de las tasas de mortalidad al tiempo que disminuyen las tasas de natalidad. Es un hecho sin precedentes y una consecuencia de la extensión a la población de los beneficios del progreso en el ámbito económico, científico, tecnológico y social. En la tabla 1 se observa que la esperanza de vida al nacimiento de las mujeres españolas es la más alta entre los quince países de la Unión Europea. Entre las de 60 y más años sólo en Francia es algo superior. Un rasgo fundamental de la vejez por las consecuencias que conlleva es que es esencialmente femenina.

Una característica del proceso de envejecimiento en España es, como en otros ámbitos también se ha producido, la rapidez con que tiene lugar. En el gráfico 1 puede verse que hasta 1950 el volumen de personas ancianas era pequeño pero comienza a ascender irremediablemente hasta alcanzar su primer pico hacia el año 2000. Otra característica del envejecimiento en las sociedades desarrolladas es el aumento del volumen y proporción de las personas más ancianas, las de 80 y más años, cuyas proyecciones para los próximos 50 años pueden observarse en la tabla 2 . Un fenómeno relacionado es el incremento notable de las personas centenarias. Entre las personas más ancianas la gran mayoría son mujeres. En el caso de España (tabla 3) la relación entre sexos más igualitaria se da entre los 35 y 44 años. Sin embargo la razón de masculinidad va disminuyendo progresivamente hasta que se inicia una diferencia cada vez más desproporcionada a partir de los 70 años. Son más las mujeres que los varones en la ancianidad, y por su mayor supervivencia sobre sus compañeros se van quedando más solas. 
Suele asociarse vejez y enfermedad, pero la gran mayoría de las personas ancianas es funcionalmente independiente y capaz de llevar una vida normal. Algunas necesitan ciertos apoyos y otras son dependientes. Lo que ocurre es que en la edad avanzada se producen pluripatologías que no es tanto por causa de la edad, cuanto por la acumulación de enfermedades y el deterioro producido en el organismo. El aumento del volumen de personas muy ancianas conlleva un aumento del número de personas con enfermedades crónicas y discapacidad.

TABLA 1. Esperanza de vida a diferentes edades. Unión Europea

\begin{tabular}{|l|c|c|c|c|c|c|}
\hline \multirow{2}{*}{ País } & \multicolumn{3}{|c|}{ Al nacer (1) } & \multicolumn{3}{c|}{ A los 60 años (2) } \\
\cline { 2 - 7 } & Varones & Mujeres & Diferencia & Varones & Mujeres & Diferencia \\
\hline UE-15 & $\mathbf{7 4 , 9}$ & $\mathbf{8 1 , 2}$ & $\mathbf{6 , 3}$ & $\mathbf{1 9 , 4}$ & $\mathbf{2 3 , 8}$ & $\mathbf{4 , 4}$ \\
\hline Alemania & 74,7 & 80,7 & 6,0 & 19,0 & 23,3 & 4,3 \\
\hline Austria & 75,1 & 81,0 & 5,9 & 19,3 & 23,6 & 4,3 \\
\hline Bélgica & 74,4 & 80,8 & 6,4 & 19,0 & 23,6 & 4,6 \\
\hline Dinamarca & 74,2 & 79,0 & 4,8 & 18,4 & 21,9 & 3,5 \\
\hline España & 75,3 & 82,5 & 7,2 & 19,8 & 24,5 & 4,7 \\
\hline Finlandia & 73,8 & 81,0 & 7,2 & 18,6 & 23,4 & 4,8 \\
\hline Francia & 74,9 & 82,4 & 7,5 & 20,0 & 25,2 & 5,2 \\
\hline Grecia & 75,5 & 80,6 & 5,1 & 20,2 & 23,1 & 2,9 \\
\hline Irlanda & 73,9 & 79,1 & 5,2 & 17,9 & 21,8 & 3,9 \\
\hline Italia & 75,6 & 82,3 & 6,7 & 19,9 & 24,2 & 4,3 \\
\hline Luxemburgo & 74,7 & 81,2 & 6,5 & 18,7 & 23,4 & 4,7 \\
\hline Países Bajos & 75,3 & 80,5 & 5,1 & 18,9 & 23,4 & 4,5 \\
\hline Portugal & 72,0 & 79,1 & 7,1 & 18,0 & 22,2 & 4,2 \\
\hline Reino Unido & 75,0 & 79,8 & 4,8 & 18,9 & 22,6 & 3,7 \\
\hline Suecia & 77,1 & 81,9 & 4,8 & 20,2 & 24,2 & 4,0 \\
\hline
\end{tabular}

(1) Datos de 1999

(2) Datos de 1998

Esperanza de vida a los 60 años: Italia1997.

Fuente: Eurostat: Statistique Sociales Européennes 2001. Luxemburgo, 2001.

(1) 174 p.; tabla G-4 y G-5, págs. 113

(2) 174 p.; tabla G-6 y G-7, págs. 114

FUENTE: http://www.imsersomayores.csic.es/

FUENTE: http://www.imsersomayores.csic.es 


\section{Envejecimiento y familia}

GRÁFICO 1. Evolución de la población mayor. España, 1900-2050 (miles)

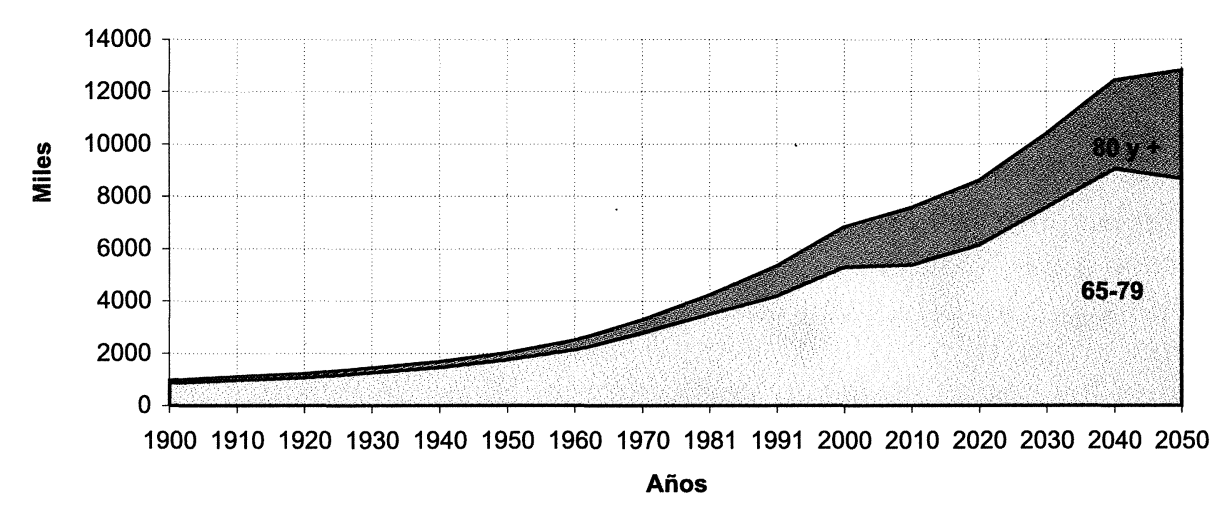

* De 1900 a 2000 los datos son reales; de 2010 a 2050 se trata de proyecciones; desde 1970 la población es de derecho.

Fuente: INE: Anuario Estadístico, varios años;

INE: Censos de Población:

INE: INEBASE: Revisión del Padrón municipal de habitantes a 1 de enero de 2000. Datos a nivel nacional, comunidad autónoma y provincia. INE, 202.

INE:INEBASE: Proyecciones de la población de España calculadas a partir del Censo Población a 31 de diciembre de 1991 INE, 2002.

Conf. Cajas de Ahorro: Estadisticas básicas de España

TABLA 2. Proporción de personas ancianas en España (1900-2050)

\begin{tabular}{|l|c|c|c|}
\hline Año & $\begin{array}{c}\text { Población } \\
\text { total }\end{array}$ & $\begin{array}{c}\text { Población 65 y } \\
\text { más }\end{array}$ & $\begin{array}{c}\text { Población 80 y } \\
\text { más }\end{array}$ \\
\hline 1900 & 18.618 .086 & $5 \%$ & $1 \%$ \\
\hline 1930 & 23.677 .794 & 6 & 1 \\
\hline 1960 & 30.528539 & 8 & 1 \\
\hline 1991 & 38.872 .268 & 14 & 3 \\
\hline 2030 & 43.369 .945 & 24 & 7 \\
\hline 2050 & 41.199 .961 & 31 & 10 \\
\hline
\end{tabular}

Fuente: http://www.imsersomayores.csic.es y elaboración propia.

Se teme el peso de la dependencia para un futuro a medio y largo plazo. El impacto en el sistema de salud del notable volumen previsto de personas ancianas será consecuencia de las condiciones de vida de la población, y del alcance de la dependencia. No se disponen de buenas medidas del bienestar físico de las personas, del bienestar relativo de los diferentes grupos de edad en la población a lo largo del tiempo. De la diversa in- 
formación existente se observa una tendencia en la mejora de los niveles de salud de las personas mayores con respecto a sus antepasadas (Jonson y Falkingham 1992: 74). Se podría pues contar en un futuro con una población en mejores condiciones que lo esperado, y la dependencia que hubiera podido preverse entre las personas mayores hace 40 años no sería de esperar en la actualidad. Las consecuencias de la mejora de las condiciones de la población anciana pueden influir en los cambios de las políticas. Con respecto a la Seguridad Social se espera que la edad de jubilación se retrase a los 70 años. Cuando a principios de siglo se establece la edad de jubilación a los 65 años, pocas personas llegaban a jubiladas, y sólo una pequeña proporción vivía el tiempo suficiente para disfrutarla. Esto supone que los costes se están posponiendo. Si no se produce ningún otro cambio puede esperarse sencillamente que el volumen de cuidados de salud que se requería anteriormente se experimentará por las personas a una edad más tardía, pero las personas necesitarán los cuidados.

TABLA 3. Estructura por sexo y edad y razón de masculinidad, 2000

\begin{tabular}{|l|r|r|r|c|}
\hline & \multicolumn{1}{|c|}{ Total } & \multicolumn{1}{c|}{ Varones } & \multicolumn{1}{c|}{ Mujeres } & $\begin{array}{c}\text { Razón de } \\
\text { masculinidad }\end{array}$ \\
\hline Total & 40.499 .790 & 19.821 .384 & 20.678 .407 & 95,9 \\
\hline $0-4$ & 1.705 .870 & 873.230 & 832.640 & 104,9 \\
\hline $5-9$ & 1.986 .754 & 1.020 .454 & 966.300 & 105,6 \\
\hline $10-14$ & 2.202 .376 & 1.130 .615 & 1.071 .760 & 105,5 \\
\hline $15-19$ & 2.689 .035 & 1.378 .525 & 1.310 .510 & 105,2 \\
\hline $20-24$ & 3.312 .843 & 1.691 .637 & 1.621 .207 & 104,3 \\
\hline $25-29$ & 3.374 .058 & 1.716 .350 & 1.657 .708 & 103,5 \\
\hline $30-34$ & 3.321 .861 & 1.678 .566 & 1.643 .294 & 102,1 \\
\hline $35-39$ & 3.185 .731 & 1.599 .151 & 1.586 .580 & 100,8 \\
\hline $40-44$ & 2.879 .096 & 1.438 .670 & 1.440 .426 & 99,9 \\
\hline $45-49$ & 2.510 .642 & 1.253 .558 & 1.257 .084 & 99,7 \\
\hline $50-54$ & 2.445 .668 & 1.209 .798 & 1.235 .869 & 97,9 \\
\hline $55-59$ & 2.130 .515 & 1.045 .527 & 1.084 .987 & 96,4 \\
\hline $60-64$ & 1.913 .198 & 912.297 & 1.000 .901 & 91,1 \\
\hline $65-69$ & 2.113 .555 & 987.743 & 1.125 .811 & 87,7 \\
\hline $70-74$ & 1.795 .404 & 799.630 & 995.775 & 80,3 \\
\hline $75-79$ & 1.387 .190 & 572.480 & 814.710 & 70,3 \\
\hline $80-84$ & 827.434 & 294.011 & 533.422 & 55,1 \\
\hline $85-89$ & 488.924 & 154.939 & 333.984 & 46,4 \\
\hline $90-94$ & 181825 & 51566 & 130260 & 39,6 \\
\hline 95 y + & 47811 & 12634 & 35177 & 35,9 \\
\hline & & & & \\
\hline
\end{tabular}

Fuente: INE: INEBASE: Revisión del Padrón municipal de habitantes a 1 de enero de 2000. INE, 2002. Fuente: http://www.imsersomayores.csic.es 
La utilización en el futuro de los cuidados de larga duración depende de las tendencias de los niveles de discapacidad de las personas ancianas, y todavía nadie sabe que ocurrirá, como variarán los modelos de morbilidad. Las tres teorías que especulan al respecto, señalan tres modelos distintos (Garber 1989: 257-259). Como las mejoras en la morbilidad han acompañado a las mejores en la esperanza de vida, se espera que el promedio en morbilidad de las personas ancianas decaiga y que la discapacidad se produzca poco antes de la muerte (Fries y Fuchs). La segunda concepción es que las personas que antes morían ahora sobreviven enfermas y discapacitadas por lo que la dependencia puede aumentar con el tiempo (Verbrugge). La tercera perspectiva (Poterba y Summers) apunta a un mantenimiento de la situación actual debido a que el incremento de la vulnerabilidad en los ancianos será compensado con las mejoras en la morbilidad debido a la medicina y otros avances. La discapacidad de las personas ancianas supervivientes del futuro depende de las condiciones específicas que mejoran por el cuidado médico. Si existe una prevención o tratamiento efectivos para los problemas de salud de los futuros ancianos pueden estar menos discapacitados que los actuales (p. 258).

Una cuestión importe es que aumentar sólo la esperanza de vida puede llevar a aumentar el porcentaje de personas ancianas dependientes. No se trata pues sólo de vivir sino de cómo se vive. Cada vez resulta más relevante conocer la esperanza de vida libre de discapacidad (EVLD). En España entre los varones de 65 y más años es de 11 años y de 12 para las mujeres de la misma edad ${ }^{1}$. Teniendo en cuenta la proporción que esos valores suponen sobre la esperanza de vida como tal, en los varones es del $70 \%$ y en las mujeres del $61 \%$. Es decir, que ellas viven más que ellos pero peor, sufriendo discapacidad. Por los distintos modelos de morbilidad, las enfermedades de los varones les llevan a morir, mientras que las de las mujeres les conducen a experimentar discapacidad.

El aumento de los costes de los cuidados de salud está causando gran preocupación social, y se convierte en un elemento de confrontación política. Se analizan los costes altos producidos en la atención de las personas con enfermedades crónicas y moribundas en las secciones de agudos donde se utiliza tecnología que es muy costosa. Se proponen cambios en el cuidado de los moribundos para que pasen del sistema de salud al de servicios sociales. A la vez se debaten los costes causados por la atención a las personas crónicas en una institución o en el entorno comunitario. Se asume que mientras las familias acepten realizar ese cuidado resultará más barato. La cuestión del análisis de los costos puede variar si todos los servicios que son prestados en la comunidad deben ser pagados. 
Las propuestas de cambio se centran en los mecanismos de financiación, la fuente de pago, o la fuente de los servicios. Una idea subyace y es que las personas ancianas deben recibir el mejor cuidado posible aunque no resulta clara ni existe consenso sobre la definición de «los mejores cuidados posibles». Un componente fundamental de los cuidados de larga duración es el cuidado informal, que son los servicios prestados por la familia y los amigos. Los costes y cantidad de esos servicios que no pasan por el mercado no se han medido adecuadamente aunque existe evidencia de que son amplios (Garber 1989: 261). En España, como en otras sociedades la gran mayoría de las personas ancianas que reciben cuidados de salud es atendida por la familia, y son mujeres las que asumen el rol de cuidadoras.

\section{Cambios familiares}

Se ha producido una revolución demográfica que tiene diversas consecuencias, pero además otros cambios que han variado las expectativas sociales sobre la vejez y la familia que existían en las sociedades industriales. El aumento de la esperanza de vida y la disminución de la fecundidad conllevan un debilitamiento de las estructuras familiares entre las diversas generaciones. Ha aumentado la diversidad en las formas familiares, normas, y costumbres. Eso conlleva que se produzca gran heterogeneidad en lo referente a la situación de las personas ancianas en sus relaciones familiares. Las personas en la actualidad están envejeciendo en familias intergeneracionales que son cuantitativa y cualitativamente diferentes de las de sus antepasadas. Ha cambiado no sólo la estructura familiar, que se ha alargado por el aumento de las generaciones y la disminución de los miembros pertenecientes a una generación, sino también la duración de los roles y relaciones familiares. En lugar de ser familias de dos o a veces tres generaciones, ahora pueden ser familias de cuatro generaciones. Además, el número de años que las personas mayores pueden ejercer sus roles familiares ha aumentado de forma significativa. Los padres pueden seguir formando parte de las vidas de sus hijos/as durante medio siglo. Como abuelos/as los lazos con sus nietos adultos, e incluso biznietos, pueden durar durante veinte años.

Otros rasgos de la revolución demográfica que han contribuido a los cambios en la vida familiar de las personas mayores incluyen variaciones en el tiempo dedicado a la procreación y crianza de los hijos. También en cuanto a la post-posición del primer hijo, y el hecho de no tener hijos. In- 
fluye igualmente el aumento de las familias monoparentales y el divorcio. Un rasgo de la nueva estructura familiar como resultado del declive de la mortalidad y fecundidad es lo que se denomina «verticalización». Significa que aumenta el número de generaciones vivas incrementándose las posibilidades de mantener relaciones intergeneracionales, al tiempo que se contraen las relaciones intra-generacionales al disminuir el número de miembros de una generación. Ese proceso tiene implicaciones en otros factores, como en la complejidad y potencialidad de las relaciones intergeneracionales y de las modalidades de convivencia multigeneracional.

Las personas en el próximo futuro envejecerán teniendo más vínculos familiares verticales que horizontales. Al tiempo que aumentan los abuelos y bisabuelos, disminuyen los hermanos, tíos y primos. Puede preguntarse si se dará un aumento semejante en los hogares multigeneracionales. Hace un siglo era probable encontrar familias de tres generaciones conviviendo en un mismo hogar. En cambio en la actualidad, aunque haya más generaciones, es más común en las sociedades contemporáneas que una persona anciana viva sola o con su cónyuge, lo que no significa que no siga manteniendo intensos lazos afectivos y emocionales con su familia (Walker, Guillemard y Alber 1993: 30).

Pueden ser varios los factores que influyen en que los miembros de una familia extensa compartan la vivienda, como pueden ser la edad, el género, el estado civil y la salud, así como la necesidad económica. Ciertas investigaciones muestran que las personas de edad tienden a vivir con una hija casada, cuando la salud, la viudez o la falta de recursos económicos hace difícil vivir a solas (Bazo 1990). En la tabla 4 se observa en España la diferencia de las formas de convivencia entre las personas ancianas en general y las de 80 y las de las de más años, así como las diferencias por género. En primer lugar se constata que las personas ancianas viven notablemente más en su propia casa que en la de otras personas, aunque entre las de más edad la proporción disminuye de forma remarcable, especialmente entre las mujeres. Algo más de la mitad de las de 80 y más años vive en otra casa (55\%) así como casi las dos quintas partes de los varones (37\%). Incluso en el conjunto de las mujeres ancianas, una cuarta parte (25\%) vive en casa de otras personas, el doble que los varones ancianos (12\%). En la convivencia en pareja la proporción de mujeres está por debajo de la de los varones, y por el contrario las mujeres viven a solas en dos veces y media la proporción de los varones, o el doble entre las personas más ancianas.

La vejez no sólo es femenina, y que para muchas mujeres significa vivir con discapacidad, sino además conlleva la pérdida de la pareja, y en 
muchos casos esto les obliga a salir de su propia casa, lugar donde se decía que «reinaban", para vivir generalmente en la de otros familiares. Vivir con hijos/as tiene consecuencias para el cuidado, y suele ser una estrategia de las familias para ocuparse de sus miembros ancianos, debido a la escasez de recursos en España en general, para atenderlas en sus domicilios o incluso en residencias.

TABLA 4. Formas de convivencia de las personas de 65 y más años en España, por género

\begin{tabular}{|l|c|c|c|c|}
\hline \multirow{2}{*}{$\begin{array}{c}\text { Modo de } \\
\text { convivencia }\end{array}$} & Total & 80 y más & Total & 80 y más \\
\cline { 2 - 5 } & $88 \%$ & $63 \%$ & $75 \%$ & $44 \%$ \\
\hline $\begin{array}{l}\text { 1-En su propia } \\
\text { casa }\end{array}$ & 7 & 10 & 18 & 19 \\
\hline 1.2- A solas & 76 & 45 & 46 & 13 \\
\hline $\begin{array}{l}\text { 1.3- Sólo con la } \\
\text { pareja o también } \\
\text { con hijos/as }\end{array}$ & 43 & 34 & 29 & 9 \\
\hline $\begin{array}{l}\text { 1.4- Sólo con la } \\
\text { pareja }\end{array}$ & 5 & 7 & 11 & 13 \\
\hline $\begin{array}{l}\text { 2- En su propia } \\
\text { casa, sin pareja, } \\
\text { con hijos/as u otros } \\
\text { familiares }\end{array}$ & 12 & 37 & 25 & 55 \\
\hline $\begin{array}{l}\text { 3- En otra casa } \\
\text { con hijos u otros } \\
\text { familiares }\end{array}$ & 2.662 & 392 & 3.253 & 704 \\
\hline Total (n) & & & & \\
\hline
\end{tabular}

Fuente: http://www.imsersomayores.csic.es y elaboración propia.

Vivir más años ha alargado el tiempo en que se detentan estatus y se representan roles familiares. Dado que las mujeres viven más que los varones, la duración de los mismos es diferente para unas y otros. Las personas en la actualidad, y sobre todo las mujeres, pueden esperar vivir al menos la mitad de su vida como hijas. Además de los estatus y roles como padres prolongados a lo largo del tiempo, también tienen lugar los que corresponden a los abuelos/as, o a la abuelidad. La prolongación de la vida permite especialmente a las mujeres, conocer a sus nietos como niños, adolescentes, jóvenes e incluso como padres y madres. Una mujer en la actualidad puede tener la probabilidad de pasar alrededor de la mitad de su vida siendo abuela. Todas estas cuestiones tienen muchas con- 
secuencias. Por ejemplo, en cuanto al cuidado familiar de las personas ancianas. Cada vez habrá más miembros de más generaciones ancianas y menos de las jóvenes. No poder compartir el cuidado de los ancianos entre hermanos/as e hijos/as supone una situación potencialmente conflictiva. Por otro lado, debido a que los roles y estatus de mujeres y varones en la estructura familiar multigeneracional aumentan en el tiempo, también surgen oportunidades nuevas para crear lazos afectivos más intensos. Hay más tiempo para compartir experiencias entre los miembros de las diversas generaciones, y la existencia de un número menor de familiares puede conllevar una facilidad mayor de implicación en el grupo familiar. La revolución demográfica experimentada ha producido un número de modelos diversos en las estructuras y roles intergeneracionales.

En cuanto al estado civil, en la ancianidad los varones tienen más probabilidad de estar casados que las mujeres, debido a las pautas diferentes de edad del matrimonio, a la mortalidad diferencial por sexos, y a la mayor tendencia de los varones con respecto a las mujeres de volver a casarse tras la viudez o divorcio. Las mujeres pueden pasar mucho tiempo de su vida como viudas. Aunque ha disminuido el número de mujeres que quedan viudas antes de los 50 años, sin embargo su mayor esperanza de vida, hace que permanezcan mucho más tiempo que antes en ese estatus. Los varones, debido a que tienden a casarse con más edad que las mujeres, y a que su esperanza de vida es más corta, tienen más probabilidad que ellas de morir antes de experimentar esa duración en los roles y estatus familiares. La viudez supone cambios profundos y pérdidas de carácter objetivo y subjetivo, teniendo consecuencias negativas en la salud y la mortalidad, aunque puede mejorar con el tiempo. Los hijos/as que suponen en general un apoyo importante para los padres/madres, lo son aún más en la viudez (Bazo 1990). Cuando las personas mayores necesitan apoyo social y cuidado lo reciben en general por este orden: del cónyuge, un hijo/a, otros familiares (INSERSO 1995a: 70). Amigos y vecinos suelen proporcionar apoyo afectivo y compañía. Los hijos/as son lo que proporcionan más apoyo instrumental, como los cuidados y atención personal y doméstica, realizando esas tareas por más tiempo.

Las personas ancianas divorciadas han sido poco estudiadas y no se conoce adecuadamente el impacto del divorcio en la ancianidad en las relaciones con hijos y nietos, ni que apoyo pueden recibir en ese momento los padres que no tuvieron la custodia legal de los hijos. Algunas personas ancianas vuelven a casarse, y otras permanecen solteras siempre. Dado que la mayoría de las personas solteras no han tenido hijos/as no pueden recibir su apoyo, por lo que son más atendidas por otros miembros de la fa- 
milia. Pero también tienen más probabilidades de institucionalización que las que tienen hijos/as (Bazo 1991). Por otro lado, el haber sido siempre solteras ha hecho que la mayor parte de las personas haya adquirido habilidades para vivir de forma independiente, y a solas, por lo que no experimentan el impacto negativo de la viudad o el divorcio, además de que tienen mejor salud física y mental que las divorciadas y viudas.

Existe una diversidad considerable en los modelos de solidaridad asociacional. Son cuatro los factores de diferenciación social, aparte de las diferencias individuales, que predicen las variaciones: género (las hijas tienden a tener una interacción más frecuente con los padres que los hijos); estado civil (la viudez incrementa los contactos y los hijos/as solteros/as mantienen un contacto más estrecho con los padres); clase social (mantienen más relación los hijos/as de las clases trabajadoras que los profesionales); y también se observan diferencias étnicas (por ejemplo, en los EEUU los hispanos mantienen los niveles de interacción más altos). En cuanto a la solidaridad que se muestra en los intercambios así como en la asistencia y apoyo, las personas mayores enfermas crónicas y discapacitadas reciben apoyo material y afectivo de sus hijos/as (Bazo 1998a, INSERSO 1995b), pero las personas mayores no son sólo receptoras de ayuda, también realizan una serie de aportaciones de carácter material, económico y afectivo a los miembros jóvenes de la familia (Bazo 1992b, 1996, OASIS ${ }^{2}$ ). De cara al futuro en sociedades envejecidas, en las que las distintas fases del ciclo vital se han alargado, donde las personas viven más años pero en mejor estado de salud que sus antepasadas, las perspectivas sobre las personas de edad como miembros que contribuyen al bienestar de la familia y también de la sociedad serán mayores.

\section{Solidaridad familiar intergeneracional}

Suele pensarse que los lazos familiares entre generaciones se han debilitado en las sociedades actuales por los cambios experimentados, pero lo que ocurre es que la familia atiende y cuida de otra manera. Por otra parte, no puede dejar de considerarse la influencia de las estructuras en el comportamiento de las personas y las familias. De esta forma, cuando los servicios provistos por el Estado son amplios y accesibles, las familias no necesitan involucrarse de la misma forma en el apoyo instrumental como cuando aquellos son escasos y poco accesibles.

Presentaré a continuación algunos resultados de una investigación que acabamos de terminar un equipo de cinco países, que ha sido financiada 


\section{Envejecimiento y familia}

por la Comisión Europea dentro del V Programa Marco. Los países son España, Alemania, Inglaterra, Noruega e Israel. El proyecto Old Age and Autonomy: The Role of Social Services Systems and Intergenerational Family Solidarity (OASIS) ha sido financiado por la Comisión Europea dentro del V Programa Marco de Investigación (Contrato número QLK6-CT-199902182). El objetivo principal del proyecto es conocer de qué manera las distintas culturas familiares y los sistemas socio-sanitarios apoyan la autonomía de las personas mayores y retrasan su dependencia, para mejorar así su calidad de vida y promover nuevas iniciativas políticas y planes de intervención. El diseño está basado en una combinación de métodos cuantitativos y cualitativos. Los datos cuantitativos se han recogido a través de un cuestionario aplicado en los cinco países sobre muestras representativas del grupo de personas de 25 a 64 años un total de 800 , y el de 75 y más años con 400 personas. En total una muestra de unas 1.200 personas en cada país, lo que supone una muestra total de 6.000 personas en los cinco países. A través del cuestionario se han identificado personas mayores en riesgo de dependencia, y se ha seleccionado una muestra de diez díadas (un total de veinte participantes en cada país) de personas mayores y su «hijo/a cuidador/a principal». Se han realizado entrevistas en profundidad, centrándose en los aspectos relacionados con la forma en que se enfrentan a la dependencia y la calidad de vida. Son entrevistas que se han analizando con el método WinMax.

En la investigación como se observa en primer lugar en la tabla 5, aparece que alrededor de algo menos de la mitad de las personas de 75 y más años de los cinco países no recibe ayuda de nadie para las tareas del hogar, el transporte y compra, y el cuidado personal. En Israel es donde más personas reciben algún tipo de apoyo. Por género, son en general las mujeres las que reciben más ayuda que los varones, sobre todo cuando se trata de recibir dos o tres tipos de ayuda a la vez.

Las personas que reciben ayuda pueden recibirla de diversas fuentes, aunque la Familia y los Servicios Públicos son las fundamentales. Cuando reciben ayuda de la familia, como se observa en la tabla 6 , donde menos se recibe es en Israel, seguido de Noruega, y donde más en Inglaterra, España y Alemania. Las mujeres son las que más apoyo reciben de la Familia. Entre quienes reciben ayuda de los Servicios (tabla 7) los países que muestran porcentajes menores en su recepción son España, Alemania e Inglaterra, y los mayores Noruega e Israel. Las mujeres reciben también más apoyo de los Servicios. Ocurre que las mujeres muestran siempre, y en la investigación también se ha detectado, niveles más bajos de capacidad funcional que los varones. Comparando las dos tablas 
Noruega e Israel son países con mayor apoyo de los Servicios sobre la Familia. El apoyo mayor de la Familia sobre los Servicios se observa por el contrario en Inglaterra, más en Alemania y sobre todo en España.

TABLA 5. Personas de 75 y más años que reciben ayuda de cualquier fuente en las tareas de la casa, transporte o compra y cuidado personal, en porcentajes

\begin{tabular}{|c|c|c|c|c|c|c|c|c|c|c|c|c|c|c|c|}
\hline & \multicolumn{3}{|c|}{ Noruega } & \multicolumn{3}{|c|}{ Inglaterra } & \multicolumn{3}{|c|}{ Alemania } & \multicolumn{3}{|c|}{ España } & \multicolumn{3}{|c|}{ Israel } \\
\hline & V & $\mathbf{M}$ & $\mathbf{T}$ & V & M & $\mathrm{T}$ & V & $\mathbf{M}$ & $\mathbf{T}$ & $\mathbf{V}$ & $\mathbf{M}$ & $\mathbf{T}$ & $\mathbf{V}$ & $\mathbf{M}$ & $\mathbf{T}$ \\
\hline Ninguno & 54 & 37 & 44 & 57 & 40 & 45 & 56 & 46 & 49 & 59 & 45 & 50 & 38 & 28 & 33 \\
\hline Uno & 26 & 24 & 25 & 17 & 17 & 17 & 13 & 14 & 13 & 24 & 24 & 24 & 28 & 27 & 28 \\
\hline Dos & 14 & 28 & 22 & 14 & 29 & 25 & 22 & 23 & 22 & 11 & 21 & 17 & 19 & 25 & 22 \\
\hline Tres & 6 & 11 & 9 & 12 & 14 & 13 & 9 & 17 & 15 & 6 & 10 & 9 & 15 & 19 & 17 \\
\hline Total & 167 & 246 & 413 & 126 & 272 & 398 & 151 & 339 & 499 & 133 & 252 & 385 & 169 & 200 & 369 \\
\hline
\end{tabular}

Nota: $\mathrm{V}=$ Varones, $\mathrm{M}=$ Mujeres, $\mathrm{T}=$ Total .

TABla 6. Personas de 75 y más años que reciben ayuda de la FAMILIA en las tareas de la casa, transporte o compra y cuidado, en porcentajes

\begin{tabular}{|c|c|c|c|c|c|c|c|c|c|c|c|c|c|c|c|}
\hline & \multicolumn{3}{|c|}{ Noruega } & \multicolumn{3}{|c|}{ Inglaterra } & \multicolumn{3}{|c|}{ Alemania } & \multicolumn{3}{|c|}{ España } & \multicolumn{3}{|c|}{ Israel } \\
\hline & V & M & $\mathbf{T}$ & V & $\mathbf{M}$ & $\mathbf{T}$ & V & $\mathbf{M}$ & $\mathbf{T}$ & V & $\mathbf{M}$ & $\mathbf{T}$ & $\mathbf{V}$ & $\mathbf{M}$ & $\mathbf{T}$ \\
\hline Ninguno & 77 & 66 & 71 & 69 & 57 & 61 & 65 & 66 & 66 & 70 & 59 & 62 & 79 & 72 & 75 \\
\hline Uno & 19 & 21 & 20 & 12 & 20 & 18 & 13 & 9 & 10 & 17 & 21 & 20 & 14 & 19 & 16 \\
\hline Dos & 3 & 11 & 8 & 12 & 18 & 16 & 15 & 17 & 16 & 7 & 13 & 11 & 5 & 6 & 5 \\
\hline Tres & 1 & 1 & 1 & 7 & 5 & 6 & 7 & 8 & 7 & 6 & 7 & 7 & 2 & 3 & 3 \\
\hline Total & 167 & 246 & 413 & 126 & 272 & 398 & 151 & 339 & 499 & 133 & 252 & 385 & 169 & 200 & 369 \\
\hline
\end{tabular}

Nota: $\mathrm{V}=$ Varones, $\mathrm{M}=$ Mujeres, $\mathrm{T}=$ Total .

Observando el compromiso e implicación de las familias en el cuidado y la atención de las personas mayores, se ha seguido profundizando en el análisis del perfil de la persona que ayuda y cuida. En la tabla 8 aparecen las respuestas dadas por las personas de la muestra que tienen algún padre o madre sobre la ayuda en las tareas de la casa. Al menos las tres cuartas partes de esas personas no ayudan a sus padres, apare- 


\section{Envejecimiento y familia}

ciendo en Alemania la proporción más alta. Ahora bien, entre quienes ayudan, las mujeres representan entre dos y tres veces (como es el caso de Israel e Inglaterra) la proporción de los varones. Siendo países los cinco, con niveles de protección social distintos así como de actividad laboral femenina, resulta interesante comprobar que las mujeres parecen seguir manteniendo niveles más altos de compromiso con el cuidado y la atención de los padres ancianos que los varones. Aún lo es más si se analizan las proporciones de mujeres empleadas en todos los países, que en España ya representan el 50\%. Todo ello tiene consecuencias en el cuidado y las políticas.

TABLA 7. Personas de 75 y más años que reciben ayuda de los SERVICIOS en las tareas de la casa, transporte o compra y cuidado personal, en porcentajes

\begin{tabular}{|c|c|c|c|c|c|c|c|c|c|c|c|c|c|c|c|}
\hline & \multicolumn{3}{|c|}{ Noruega } & \multicolumn{3}{|c|}{ Inglaterra } & \multicolumn{3}{|c|}{ Alemania } & \multicolumn{3}{|c|}{ España } & \multicolumn{3}{|c|}{ Israel } \\
\hline & V & M & $\mathbf{T}$ & V & $\mathbf{M}$ & $\mathbf{T}$ & V & $\mathbf{M}$ & $\mathbf{T}$ & V & $\mathbf{M}$ & $\mathbf{T}$ & $\mathbf{V}$ & M & $\mathbf{T}$ \\
\hline Ninguno & 68 & 51 & 58 & 82 & 72 & 75 & 92 & 81 & 84 & 95 & 91 & 93 & 70 & 65 & 67 \\
\hline Uno & 22 & 29 & 26 & 15 & 17 & 16 & 1 & 6 & 4 & 4 & 4 & 4 & 15 & 17 & 16 \\
\hline Dos & 5 & 13 & 10 & 2 & 7 & 5 & 4 & 5 & 5 & 1 & 4 & 4 & 8 & 5 & 6 \\
\hline Tres & 5 & 7 & 6 & 2 & 5 & 4 & 3 & 7 & 7 & 0 & 1 & 1 & 6 & 12 & 9 \\
\hline Total & 167 & 246 & 413 & 126 & 272 & 398 & 151 & 339 & 499 & 133 & 252 & 385 & 169 & 200 & 369 \\
\hline
\end{tabular}

Nota: $\mathrm{V}=$ Varones, $\mathrm{M}=$ Mujeres, $\mathrm{T}=$ Total .

TABLA 8. Personas que ayudan a sus padres en las tareas de la casa, por género y situación laboral, en porcentajes

\begin{tabular}{|l|c|c|c|c|c|}
\hline & Noruega & Inglaterra & Alemania & España & Israel \\
\hline No ayudan & 76 & 75 & 83 & 75 & 77 \\
\hline Ayudan & 24 & 25 & 17 & 25 & 23 \\
\hline Hombres & 33 & 26 & 23 & 32 & 26 \\
\hline Mujeres & 67 & 74 & 77 & 68 & 74 \\
\hline Hombres con trabajo & 79 & 67 & 69 & 61 & 73 \\
\hline Mujeres con trabajo & 70 & 65 & 66 & 50 & 65 \\
\hline Amas de casa & 6 & 20 & 21 & 21 & 10 \\
\hline Total & 543 & 367 & 409 & 468 & 551 \\
\hline
\end{tabular}

Las personas mayores son receptoras de cuidados, ayuda, apoyo, pero también «donadoras». Entre las generaciones se produce un intercambio 
de ayuda, bienes, servicios, apoyos. Analizaremos brevemente algunos de esos intercambios entre la generación de padres y la de hijos. Las personas de la muestra que tienen hijos/as (tabla 9) manifiestan recibir ayuda de ellos en diversos aspectos, con proporciones distintas entre los cinco países. En el apoyo emocional es donde se observan proporciones más altas. Si nos fijamos en el apoyo económico, en España, Inglaterra e Israel se dan las proporciones más altas de padres/madres que manifiestan recibir ayuda económica de sus hijos/as, aunque no resulten importantes. Una ayuda fundamental para el mantenimiento de las personas ancianas en su hogar es la aportada para la realización de las tareas domésticas. España es de los cinco países donde más se manifiesta recibir esa ayuda (33\%).

TABLA 9. Ayuda recibida de hijos

\begin{tabular}{|l|c|c|c|c|c|c|c|c|c|c|}
\hline & \multicolumn{2}{|c|}{ Noruega } & \multicolumn{2}{|c|}{ Inglaterra } & \multicolumn{2}{c|}{ Alemania } & \multicolumn{2}{c|}{ España } & \multicolumn{2}{c|}{ Israel } \\
\hline $\begin{array}{l}\text { Ayuda en reparaciones } \\
\text { domésticas 0 jardín }\end{array}$ & 229 & $35 \%$ & 257 & $32 \%$ & 263 & $37 \%$ & 159 & $23 \%$ & 114 & $15 \%$ \\
\hline $\begin{array}{l}\text { Ayuda en transporte 0 } \\
\text { compra }\end{array}$ & 238 & 37 & 358 & 44 & 271 & 38 & 234 & 33 & 217 & 29 \\
\hline Ayuda en tareas domésticas & 116 & 18 & 217 & 27 & 203 & 29 & 229 & 33 & 126 & 17 \\
\hline $\begin{array}{l}\text { Ayuda en cuidado } \\
\text { personal }\end{array}$ & 11 & 2 & 52 & 7 & 68 & 10 & 51 & 7 & 28 & 4 \\
\hline Apoyo Económico & 20 & 3 & 77 & 10 & 29 & 4 & 75 & 11 & 66 & 9 \\
\hline Apoyo emocional & 306 & 47 & 432 & 54 & 384 & 54 & 422 & 60 & 440 & 59 \\
\hline $\begin{array}{l}\text { Número total de } \\
\text { personas con hijos adultos }\end{array}$ & \multicolumn{2}{|c|}{656} & \multicolumn{2}{|c|}{807} & 717 & 701 & 750 \\
\hline
\end{tabular}

Si se analiza la regularidad con que se produce esa ayuda, se observa que la ayuda económica que los padres/madres dicen recibir de sus hijos/as (tabla 10) es de carácter eminentemente ocasional y no regular en Noruega, Israel y Alemania. Sin embargo en Inglaterra y sobre todo en España es más bien realizada de forma regular. Ocurre con la ayuda prestada en las tareas domésticas. En España y en menor proporción en Inglaterra las familias se ven obligadas a ayudar en la casa a sus padres/madres de manera regular, sobre todo en España donde sólo la cuarta parte de las personas que tienen algún hijo/a declara que la ayuda recibida lo es de forma ocasional. 
Envejecimiento y familia

TABLA 10. Porcentajes de la ayuda recibida de hijos de forma ocasional

\begin{tabular}{|l|c|c|c|c|c|c|c|c|c|c|}
\hline & \multicolumn{2}{|c|}{ Noruega } & \multicolumn{2}{|c|}{ Inglaterra } & \multicolumn{2}{c|}{ Alemania } & \multicolumn{2}{c|}{ España } & \multicolumn{2}{c|}{ Israel } \\
\hline $\begin{array}{l}\text { Ayuda en reparaciones } \\
\text { domésticas 0 jardín }\end{array}$ & 130 & $73 \%$ & 149 & $62 \%$ & 188 & $75 \%$ & 69 & $45 \%$ & 63 & $56 \%$ \\
\hline $\begin{array}{l}\text { Ayuda en transporte 0 } \\
\text { compra }\end{array}$ & 112 & 63 & 139 & 42 & 136 & 52 & 74 & 33 & 115 & 54 \\
\hline Ayuda en tareas domésticas & 55 & 64 & 80 & 40 & 96 & 51 & 61 & 27 & 65 & 52 \\
\hline $\begin{array}{l}\text { Ayuda en cuidado } \\
\text { personal }\end{array}$ & 6 & 60 & 13 & 33 & 37 & 56 & 5 & 10 & 13 & 50 \\
\hline Apoyo Económico & 11 & 69 & 32 & 48 & 25 & 86 & 22 & 31 & 39 & 61 \\
\hline Apoyo emocional & 126 & 53 & 189 & 46 & 242 & 66 & 108 & 26 & 149 & 34 \\
\hline $\begin{array}{l}\text { Número total de } \\
\text { personas con hijos adultos }\end{array}$ & 656 & \multicolumn{2}{|c|}{807} & 717 & 701 & 750 \\
\hline
\end{tabular}

TABLA 11. Ayuda recibida de padres

\begin{tabular}{|c|c|c|c|c|c|c|c|c|c|c|}
\hline \multirow[b]{2}{*}{$\begin{array}{l}\text { Ayuda en reparaciones } \\
\text { domésticas } 0 \text { jardín }\end{array}$} & \multicolumn{2}{|c|}{ Noruega } & \multicolumn{2}{|c|}{ Inglaterra } & \multicolumn{2}{|c|}{ Alemania } & \multicolumn{2}{|c|}{ España } & \multicolumn{2}{|c|}{ Israel } \\
\hline & 128 & $23 \%$ & 39 & $10 \%$ & 34 & $8 \%$ & 37 & $8 \%$ & 67 & $12 \%$ \\
\hline $\begin{array}{l}\text { Ayuda en transporte } 0 \\
\text { compra }\end{array}$ & 125 & 23 & 39 & 10 & 20 & 5 & 66 & 14 & 77 & 14 \\
\hline Ayuda en tareas domésticas & 91 & 17 & 34 & 9 & 40 & 10 & 78 & 17 & 62 & 11 \\
\hline $\begin{array}{l}\text { Ayuda en cuidado } \\
\text { personal }\end{array}$ & -- & -- & 1 & -- & 7 & 2 & 3 & 1 & 8 & 1 \\
\hline $\begin{array}{l}\text { Ayuda en el cuidado } \\
\text { de los hijos* }\end{array}$ & 164 & 30 & -- & -- & 72 & 17 & 50 & 11 & 128 & 23 \\
\hline Apoyo Económico & 193 & 35 & 63 & 16 & 68 & 16 & 77 & 16 & 409 & 73 \\
\hline Apoyo emocional & 324 & 59 & 180 & 47 & 234 & 56 & 237 & 50 & 440 & 78 \\
\hline $\begin{array}{l}\text { Número total de } \\
\text { personas con algún padre } 0 \\
\text { madre }\end{array}$ & \multicolumn{2}{|c|}{550} & \multicolumn{2}{|c|}{385} & \multicolumn{2}{|c|}{423} & \multicolumn{2}{|c|}{476} & \multicolumn{2}{|c|}{564} \\
\hline
\end{tabular}

* Datos no disponibles para Inglaterra

Si son los hijos/as los que responden sobre las ayudas recibidas de los padres (tabla 11) también se aprecia el apoyo de estos en proporciones diferentes. En todos los países entre las personas de la muestra que tienen algún padre o madre se declara recibir apoyo económico de estos, destacando Israel donde el $73 \%$ de los hijos afirma recibir esta ayuda. Notablemente más baja, pero una tercera parte en Noruega, también manifiesta recibir ayuda económica. En los otros tres países son menos quienes reci- 
ben ayuda y, curiosamente, aparece la misma proporción (16\%). En todos los países hay más hijos que dicen recibir ayuda económica de los padres que viceversa. En porcentajes menores en su conjunto, pero también reciben ayuda de los padres/madres en las tareas domésticas, con las proporciones más altas en Noruega y España (17\% en ambos países). En la ayuda en el cuidado de los niños/as el país con la proporción más alta de personas que declara recibir ayuda de los padres/madres es Noruega, seguido de Israel, siendo España el país con menor proporción de personas que declara recibir ayuda de sus padres en el cuidado de los hijos.

En cuanto a la frecuencia en la ayuda recibida por las personas que tienen algún padre/madre (tabla 12) en las tareas domésticas en Noruega y Alemania esa ayuda es mayoritariamente ocasional, seguido de Inglaterra con casi la mitad de los casos. Mientras tanto, en España y mayoritariamente en Israel, la ayuda se presta de forma regular, aunque en Israel sólo aparecen cuatro personas. El apoyo económico que dicen recibir de sus padres, excepto en España, también es mayoritariamente de forma ocasional llegando a ser casi la totalidad de los casos en Noruega. El cuidado de los nietos/as también excepto en Israel es proporcionado mayoritariamente de forma ocasional. Comparando España con Noruega si en la frecuencia de ayuda en España se declaraba recibir una proporción de ayuda la tercera parte que en Noruega, sin embargo, en España se recibe esa ayuda de forma más regular (49\%). Ayudan menos cantidad de abuelos/as pero ayudan de forma más intensiva. Algo que también parece que sucede en Israel.

TABLA 12. Porcentajes de la ayuda recibida de padres de forma ocasional

\begin{tabular}{|c|c|c|c|c|c|c|c|c|c|c|}
\hline \multirow[b]{2}{*}{$\begin{array}{l}\text { Ayuda en reparaciones } \\
\text { domésticas o jardín }\end{array}$} & \multicolumn{2}{|c|}{ Noruega } & \multicolumn{2}{|c|}{ Inglaterra } & \multicolumn{2}{|c|}{ Alemania } & \multicolumn{2}{|c|}{ España } & \multicolumn{2}{|c|}{ Israel } \\
\hline & 103 & $83 \%$ & 34 & 92 & 30 & $86 \%$ & 18 & $50 \%$ & 16 & $32 \%$ \\
\hline $\begin{array}{l}\text { Ayuda en transporte } 0 \\
\text { compra }\end{array}$ & 114 & 92 & 25 & 68 & 15 & 71 & 25 & 40 & 34 & 56 \\
\hline Ayuda en tareas domésticas & 78 & 86 & 15 & 48 & 30 & 77 & 21 & 29 & 4 & 9 \\
\hline $\begin{array}{l}\text { Ayuda en cuidado } \\
\text { personal }\end{array}$ & -- & -- & -- & -- & 2 & 40 & -- & -- & - & -- \\
\hline $\begin{array}{l}\text { Ayuda en el cuidado } \\
\text { de los hijos }\end{array}$ & 124 & 76 & -- & -- & 50 & 70 & 25 & 51 & 26 & 33 \\
\hline Apoyo Económico & 171 & 91 & 47 & 78 & 58 & 84 & 35 & 49 & 71 & 52 \\
\hline Apoyo emocional & 250 & 78 & 48 & 13 & 162 & 70 & 74 & 32 & 61 & 30 \\
\hline $\begin{array}{l}\text { Número total de } \\
\text { personas con algún padre } 0 \\
\text { madre }\end{array}$ & \multicolumn{2}{|c|}{550} & \multicolumn{2}{|c|}{385} & \multicolumn{2}{|c|}{423} & \multicolumn{2}{|c|}{476} & \multicolumn{2}{|c|}{564} \\
\hline
\end{tabular}


Diversos aspectos han sido analizados y se han constatado las similitudes y diferencias entre países y dentro de cada país por grupos de edad. Los dos países que se han observado más opuestos de los cinco tanto en comportamientos como en valores y preferencias, son Noruega y España. Los otros tres tienden a mantener posturas más intermedias. Israel se parece a Noruega en cuanto a la provisión de los servicios, pero es más familista. Alemania e Inglaterra tienden a ocupar posiciones intermedias. Además Noruega y España difieren notablemente en cuanto a los sistemas formales de bienestar.

Se analiza a continuación algunos resultados comparativos entre esos dos países obtenidos en algunas preferencias, valores y opiniones sobre las responsabilidades atribuibles al Estado y/o a la Familia. Respecto a las preferencias sobre por cual institución desearían ser atendidos en caso de necesidad, como se observa en la tabla 13 en Noruega se prefieren claramente los servicios públicos, y en el otro extremo se encuentra España. Los encuestados españoles prefieren de forma mayoritaria ser cuidados por la familia.

TABLA 13. Preferencias para el cuidado en caso de necesidad

\begin{tabular}{|c|c|c|}
\hline Institución & $\begin{array}{c}\text { Noruega } \\
(\%)\end{array}$ & $\begin{array}{c}\text { España } \\
(\%)\end{array}$ \\
\hline Familia & 18 & 68 \\
\hline Servicios & 77 & 31 \\
\hline $\mathrm{N}$ & 1152 & 1092 \\
\hline
\end{tabular}

En cuanto a que miembro de la familia se prefiere, en casi todos los países se muestran como preferidos tres miembros, cónyuge, hija, e hijo. Los porcentajes para los restantes miembros de la familia son en general insignificantes. En España la opción preferida es la hija (60\%) seguida del cónyuge (52\%) y del hijo (50\%). Nos encontramos con modelos de preferencias diferentes según países. En un extremo Noruega podría ser denominado "welfarista», mientras en el otro extremo España sería «familista». (En el grupo de los cinco países, Israel se parece más en este tipo de preferencias a Noruega, Alemania e Inglaterra se mantienen en una posición intermedia).

Los resultados sobre los valores manifestados acerca de que institución es la responsable de hacer frente a ciertas responsabilidades aparecen en la tabla 14. Esas responsabilidades son en la provisión de apoyo 
económico, ayuda en las tareas domésticas, y apoyo en la atención personal. Parece que se mantiene la distinta tendencia observada en los dos países en cuanto las preferencias, ahora en una valoración mayor de los servicios prestados por el Estado con relación a los prestados por la familia en el caso de Noruega en comparación con España. Incluso en la provisión económica, en España a pesar de la extensión del sistema de pensiones a la población anciana, se sigue considerando responsabilidad importante de la familia, aunque en proporciones menores que los otros dos tipos de responsabilidad.

TABLA 14. Valores sobre responsabilidades hacia las personas ancianas

\begin{tabular}{|l|c|c|c|c|c|c|}
\hline \multirow{2}{*}{ Institución } & \multicolumn{3}{|c|}{ Noruega } & \multicolumn{3}{c|}{ España } \\
\cline { 2 - 7 } & $1(\%)$ & $2(\%)$ & $3(\%)$ & $1(\%)$ & $2(\%)$ & $3(\%)$ \\
\hline Totalmente Familia & -- & 1 & 1 & 2 & 5 & 7 \\
\hline Principalmente familia & 5 & 7 & 5 & 13 & 24 & 27 \\
\hline $\begin{array}{l}\text { Tanto el Estado como la } \\
\text { Familia }\end{array}$ & 17 & 22 & 16 & 35 & 35 & 34 \\
\hline Principalmente el Estado & 50 & 50 & 52 & 33 & 23 & 20 \\
\hline Totalmente el Estado & 27 & 18 & 25 & 17 & 13 & 11 \\
\hline No consta & 2 & 1 & 1 & -- & -- & -- \\
\hline N & 1199 & 1199 & 1199 & 1168 & 1170 & 1168 \\
\hline
\end{tabular}

1. Responsabilidad para el apoyo económico

2. Responsabilidad para la ayuda en las tareas domésticas

3. Responsabilidad para la ayuda en el cuidado personal

En la tabla resumen 15 se muestra de forma más patente las tendencias observadas. Se confirma la tendencia welfarista en Noruega, y la familista en España. En los tres tipos de responsabilidades la tendencia en España con respecto a Noruega es notablemente más familista. En Noruega sólo en la responsabilidad en la ayuda en las tareas domésticas, la proporción que entiende que debe ser compartida entre el Estado y la Familia llega a la quinta parte. En España la proporción más baja en cuanto a la responsabilidad única de la familia se observa en el apoyo económico, con un $15 \%$ de acuerdo. Las décadas de prestación de pensiones entre las personas ancianas ha llevado a la conciencia en nuestro país de que tal responsabilidad es eminentemente pública, aunque una tercera parte entiende que debe ser compartida entre el Estado y la Familia.

Por último y respecto a las opiniones sobre la responsabilidad del cuidado a las personas ancianas, pero ahora de cara al futuro, como se ob- 
serva en la tabla 16 destaca una tendencia en España hacia una aproximación al modelo «welfarista». Si se compara esta tabla con la tabla 13 se observa ahora que en Noruega se acentúa más la tendencia a que sea el Estado quien cuide y proteja a las futuras personas ancianas. España es el país con una proporción menor en la respuesta sobre la responsabilidad del Estado. En todos los países aumentan los porcentajes de la responsabilidad del Estado de cara al cuidado de las personas ancianas en el futuro, pero en comparación con la tabla 13 España es el país que aumenta más ese porcentaje (27 puntos).

TABLA 15. Tabla resumen sobre valores de las responsabilidades para las personas ancianas

\begin{tabular}{|l|c|c|c|}
\hline \multicolumn{2}{|c|}{ Países } & Noruega & España \\
\hline $\begin{array}{l}\text { Responsabilidad para el } \\
\text { apoyo económico }\end{array}$ & Más familia & 5 & 15 \\
\cline { 2 - 4 } $\begin{array}{l}\text { Responsabilidad para la } \\
\text { ayuda en las tareas } \\
\text { domésticas }\end{array}$ & F=E & 17 & 35 \\
\cline { 2 - 4 } & Más familia & 8 & 29 \\
\hline $\begin{array}{l}\text { Responsabilidad para el } \\
\text { apoyo en el cuidado } \\
\text { personal }\end{array}$ & F=E & 22 & 35 \\
\cline { 2 - 4 } & Más Familia & 6 & 34 \\
\hline
\end{tabular}

TABLA 16. Opiniones acerca de la responsabilidad en el futuro del cuidado de las personas ancianas

\begin{tabular}{|l|c|c|}
\hline $\begin{array}{c}\text { Responsabilidad de } \\
\text { cada institución }\end{array}$ & Noruega & España \\
\hline Familia & $6 \%$ & $38 \%$ \\
\hline Servicios Públicos & 90 & 58 \\
\hline $\begin{array}{l}\text { Organizaciones } \\
\text { Voluntarias }\end{array}$ & 1 & 2 \\
\hline Sector Privado & 3 & 1 \\
\hline Otros & -- & -- \\
\hline TOTAL & 1168 & 1071 \\
\hline
\end{tabular}

Finalmente, señalar que en los estudios sobre la familia realizados en España tiende a verse el lado armonioso de las relaciones familiares y no 
tanto el posible conflicto existente. En el proyecto OASIS se ha explorado también tanto el conflicto como la ambivalencia en las relaciones entre padres e hijos. En la tabla 17 con datos de España se observa que la existencia manifestada de conflicto es pequeña, aunque sea mínimamente mayor con el padre que con la madre, y aumente algo cuando se produce una situación de cuidado, sobre todo con el padre.

TABLA 17. Conflicto entre padres e hijos (desde la perspectiva de estos últimos) sin necesidad de cuidado de los padres y con necesidad de cuidado (España)

\begin{tabular}{|l|c|c|c|c|}
\hline $\begin{array}{c}\text { Existencia de } \\
\text { conflicto o } \\
\text { tensión }\end{array}$ & Nada \% & Poco \% & Algo \% & Total (n) \\
\hline Tiene padre & $54 \%$ & $30 \%$ & $12 \%$ & 328 \\
\hline Tiene madre & 58 & 28 & 9 & 447 \\
\hline $\begin{array}{l}\text { Tiene padre } \\
\text { dependiente }\end{array}$ & 46 & 41 & 10 & 123 \\
\hline $\begin{array}{l}\text { Tiene madre } \\
\text { dependiente }\end{array}$ & 52 & 32 & 11 & 187 \\
\hline
\end{tabular}

En todos los países se observa una tendencia similar, es decir niveles bajos de conflicto expresado. Se observa que los lazos de afecto son estrechos especialmente en Israel, y curiosamente notablemente más bajos que el conjunto en Alemania. También se observa que los hijos manifiestan niveles de conflicto más altos que los padres. En cuanto a la ambivalencia la tendencia en los cinco países es similar, aunque en Alemania se observan ciertas tensiones intergeneracionales manifestadas tanto por los padres como por los hijos. Son resultados que muestran claramente más afecto que conflicto, pero que también apuntan a la necesidad de proveer de apoyo exterior a las familias que cuidan de personas ancianas dependientes, a fin de evitar posibles malos tratos y sobre todo mejorar la calidad de vida de todos sus miembros.

\section{Conclusiones}

De forma breve destacaría entre otras las siguientes conclusiones. Entre las generaciones se produce un intercambio de apoyo, bienes y ayuda. A mayor extensión de los servicios prestados por el Estado se necesi- 
ta menor aportación de la familia. Dicho esto de forma genérica otra consideración es que en general las personas tienden a apoyarse mutuamente, lo que ocurre es que los tipos de ayuda y la regularidad con que se produce varía en función de las necesidades de cada generación. Los hijos son más ayudados económicamente que los padres. Varía también entre países en función de condiciones estructurales y culturales. Así, en España se produce menos ayuda de los abuelos/as en el cuidado de los nietos/as pero es más intensa. Del mismo modo es baja la proporción de ayuda económica de los hijos a los padres, pero debe prestarse de forma constante.

Respecto a las preferencia y valores mostrados se observan diferencias estructurales entre países. Noruega representaría el modelo welfarista y España el familista. Israel tiende a parecerse a Noruega aunque otorga más responsabilidad a la familia, en Alemania se observa una tendencia mixta. Los diferentes Estados del Bienestar existentes en los cinco países, así como factores culturales influyen en las manifestaciones de los encuestados. Sin embargo, es interesante destacar que otra tendencia se observa y es la idea de que en el futuro la responsabilidad sobre el cuidado y asistencia de las personas ancianas debe recaer principalmente en los Servicios, de los que en casi todos los países son sobre todo Públicos, es decir, provistos por el Estado. En Alemania se produce una excepción en este aspecto, pues se da una relación muy equilibrada entre los servicios públicos, los comerciales, y los provistos por asociaciones voluntarias.

En España resulta especialmente interesante subrayar el cambio generacional observado a este respecto. Se produce un acercamiento a los valores e ideales de los otros países con Estados de bienestar más desarrollados. Sin embargo, dado que los gobiernos actualmente tienden a acentuar más la responsabilidad familiar e individual o incluso comunitaria en detrimento de la del Estado al menos en el sentido clásico, queda por ver el papel que jugarán los ciudadanos europeos en las decisiones de los gobiernos sobre las políticas públicas.

\section{Referencias bibliográficas}

BAzo, M. T. (1990) La sociedad anciana (1990) Madrid: Centro de Investigaciones Sociológicas. Siglo XXI.

Bazo, M. T. (1992) «La ancianidad a través de los/as jóvenes», Papers. Revista de Sociología, 40, pp. 57-73.

BAzo, M. T. (1996) «Aportaciones de las personas mayores a la sociedad: Análisis sociológico», Revista Española de Investigaciones Sociológicas, 73: 209-222.

BAzo, M. T. (1990) «Institucionalización de personas ancianas: Un reto sociológico» $R e$ vista Española de Investigaciones Sociológicas, 53, pp. 149-164. 
BAZO, M. T. «El cuidado familiar en las personas ancianas con enfermedades crónicas: El caso de los pacientes con enfermedad de Alzheimer» (1998) Revista Española de Geriatría y Gerontología, 33 (1): 49-56.

GARBER, A. M. (1989) «Long-term care, wealth, and health of the disabled elderly» en David A. Wise ed. The Economics of Aging. Chicago: The National Bureau of Economic Research: 257-277.

INSERSO (1995a) Las personas mayores en España. Perfiles. Reciprocidad familiar. Madrid: Ministerio de Asuntos Sociales.

INSERSO (1995b) Cuidados en la vejez: El apoyo informal. Madrid: Ministerio de Asuntos Sociales.

Johnson, P. y J. FALKInGHAM (1992) Ageing and Economic Welfare. Londres: SAGE.

Walker, A., A. M. GuIllemard y J. AlBer (1993) Older People in Europe: Social and Economic Policies: The 1993 Report of the European Community Observatory. Luxemburgo: Comisión de las Comunidades Europeas.

\section{Notas}

$1 \mathrm{http}: / / w w w . i m s e r s o m a y o r e s . c s i c . e s$

2 Old Age and Autonomy: The Role of Social Services Systems and Intergenerational Family Solidarity (OASIS) es el proyecto europeo cuyo desarrollo explico y sobre cuyos datos presento algunos resultados. 